\section{Zum Abklingen der aus KJ und KJ(In) ausgeleuchteten Eigenlumineszenz nach Erregung durch Röntgen-Bestrahlung}

Gerhard Stühmer, Hartwig Blume und Peter Brauer

Physikalisches Institut der Universität Freiburg i. Br.

(Z. Naturforschg. 22 a, 1811-1813 [1967] ; eingeg. am 14. September 1967)

Die bei Temperaturen unterhalb etwa $120^{\circ} \mathrm{K}$ beobachtbare $371 \mathrm{~m} \mu$-Eigenlumineszenz des $\mathrm{KJ}$ kann auf folgende Arten erzeugt werden: 1. Durch optische Erregung in der Grundgitterabsorption ${ }^{1,2}$, und zwar a) im Bereich des 1. Excitonenabsorptionsmaximums und b) im Bereich der Band - Band-Übergänge, 2. nach Erregung durch RöNTGEN-Strahlen unterhalb $100{ }^{\circ} \mathrm{K}$ aus Kristallen mit Elektronenhaftstellen durch Ausleuchten mit rotem oder infrarotem Licht ${ }^{3-6}$, und 3. durch Erregung mit energiereicher ionisierender Strahlung ${ }^{3-5,7}$.

Die Emissionsspektren sind bei den vier Erregungsarten ähnlich, jedoch nicht identisch. $\mathrm{Ob}$ die Lumineszenz in allen Fällen - nach Ablauf vorhergehender Teilprozesse - dem gleichen elektronischen Übergang entspricht, könnte aus Messungen der Lumineszenzabklingdauern mit entschieden werden.

Abb. 1 zeigt noch einmal unsere Ergebnisse an $\mathrm{KJ}$ und $\mathrm{KJ}(\mathrm{Tl})$ bei optischer Erregung - in guter Übereinstimmung mit Fröhlichs Resultaten ${ }^{8,9}$. Ahrenkiel ${ }^{10}$ fand qualitativ ähnliches Temperaturverhalten der $\mathrm{Ab}$ klingzeit der $\mathrm{V}_{\mathrm{K}}$-Rekombinationslumineszenz bei Ausleuchten von $\mathrm{KJ}(\mathrm{Tl})$, nicht jedoch bei Ausleuchten von reinem durch RöNTGEN-Bestrahlung verfärbten KJ: hier nahm bei wachsender Temperatur die Abklingdauer zu und durchlief ein Maximum.

Wir berichten über den Versuch einer Aufklärung dieses Verhaltens durch Messung des Abklingens der ausgeleuchteten $\mathrm{V}_{\mathrm{K}}$-Lumineszenz an unverfärbten und verfärbten $\mathrm{KJ}(\mathrm{In})$-Kristallen. In an Stelle von Tl ermöglicht eine bessere spektroskopische Abtrennung der $371 \mathrm{~m} \mu$-Lumineszenz.

\section{Experimentelles}

Kristalle: $\mathrm{KJ}$ reinst (von $\mathrm{K}$. $\mathrm{K}$ o $\mathrm{r} \mathrm{th}$, Kiel); $\mathrm{KJ}$ (In) nach KYropoulos in reduzierender Atmosphäre mit In $/ \mathrm{K}=10^{-3}$ in der Schmelze. - Röntgen-Erregung: $50 \mathrm{kV}, 15 \mathrm{~mA}$. - Ausleuchtung der Lumineszenz mit Lichtblitzen: $0,6<\lambda<1,2 \mu$ bei $\mathrm{KJ}$ reinst, $\lambda>0,85 \mu$ bei $\mathrm{KJ}(\mathrm{In})$. - Blitzdaten: 34 nsec Halbwertsdauer, $400 \mathrm{nsec}$ nach Zündung waren 95\% der Gesamtleistung emittiert. - Auswertung der Abklingkurvenaufnahmen ab etwa 200 nsec nach Zündung.

1 M. Tomura u. J. Kalfu, J. Phys. Soc. Japan 15, 1295 [1960].

2 J. Ramamurti u. K. Teegarden, Phys. Rev. 145, 698 [1966].

3 M. N. Kabler, Phys. Rev. 136, A 1296 [1964].

4 R. B. Murray u. F. J. Keller, Phys. Rev. 137, A 942 [1965].

5 R. G. Kaufman u. W. B. Hadley, J. Chem. Phys. 44, 1311 [1966].

6 W. B. Hadley, S. Polick, G. Kaufman u. H. N. Hersh, J. Chem. Phys. 45, 2040 [1966].

\section{Ergebnisse}

1. Aus $\mathrm{KJ}(\mathrm{In})$ ist nach Röntgen-Bestrahlung (10 Min.) unterhalb $100{ }^{\circ} \mathrm{K}$ mit infrarotem Licht $(\lambda>850$ $\mathrm{m} \mu$ ) Rekombinationslumineszenz ausleuchtbar, die exponentiell abklingt. Während der Ausleuchtung konnte Photoleitung beobachtet werden.

2. Die Abklingdauern $\tau$ stimmen im ganzen gemessenen Temperaturbereich innerhalb des Meßfehlers mit denen bei Erregung in der Grundgitterabsorption ${ }^{8,9}$ überein.

3. Aus durch RöNTGEN-Bestrahlung verfärbtem KJ (RöntGen-Erregung: bis zu 30 Min. bei $140^{\circ} \mathrm{K}$, anschließend 15 Min. bei $80{ }^{\circ} \mathrm{K}$ ) und $\mathrm{KJ}(\mathrm{In})$ (RöntgenErregung: bis zu 20 Min. bei $140{ }^{\circ} \mathrm{K}, 15$ Min. bei $80^{\circ} \mathrm{K}$ ) ausgeleuchtete Lumineszenz klingt nicht mehr exponentiell ab. Die Zeit $\vartheta$ für den Intensitätsabfall vom Maximum bis auf $1 / e$ ist stets länger als die unter

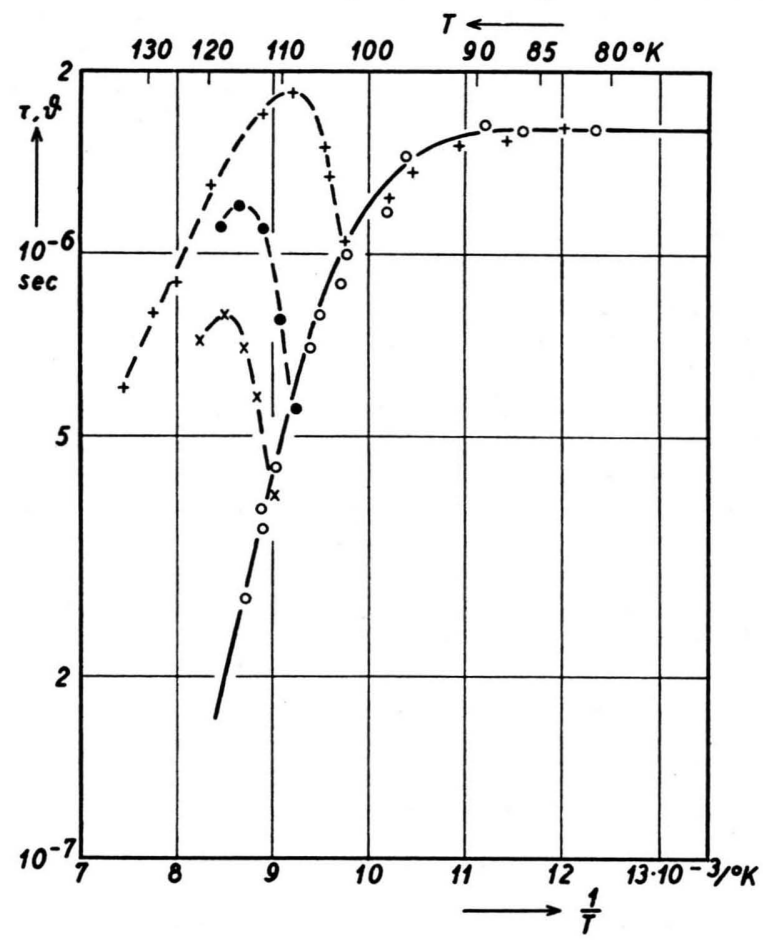

Abb. 1. Abklingdauer $\tau$ bzw. Abklingzeit $\vartheta$ der $371 \mathrm{~m} \mu$-Lumineszenz in Abhängigkeit von der Temperatur. a) Bei optischer Erregung: - $\mathrm{KJ}$ und $\mathrm{KJ}(\mathrm{Tl})$ nach Ref. ${ }^{8}$; b) bei Ausleuchtung: $\bigcirc-\circ \mathrm{KJ}(\mathrm{In})$ unverfärbt, $+\longrightarrow+\mathrm{KJ}$ verfärbt durch Röntgen-Bestrahlung, $\times-\times \mathrm{KJ}$ (In) verfärbt $(10 \mathrm{Min}$. RöNtgen-Bestrahlung bei $\left.140^{\circ} \mathrm{K}\right),-\longrightarrow \mathrm{KJ}(\mathrm{In})$ verfärbt (20 Min. Röntgen-Bestrahlung bei $140^{\circ} \mathrm{K}$ ).

7 R. Fieschi u. G. Spinolo, Nuovo Cimento 23, 738 [1962].

8 H. Blume, P. Brauer u. G. Stühmer, Z. Naturforschg. 21 a, 849 [1966].

9 D. Fröhlich, Bull. Am. Phys. Soc. Ser. II, 11, 87 [1966] und persönliche Mitteilung.

10 R. K. Ahrenkiel, Solid State Commun. 4, 21 [1966]. 


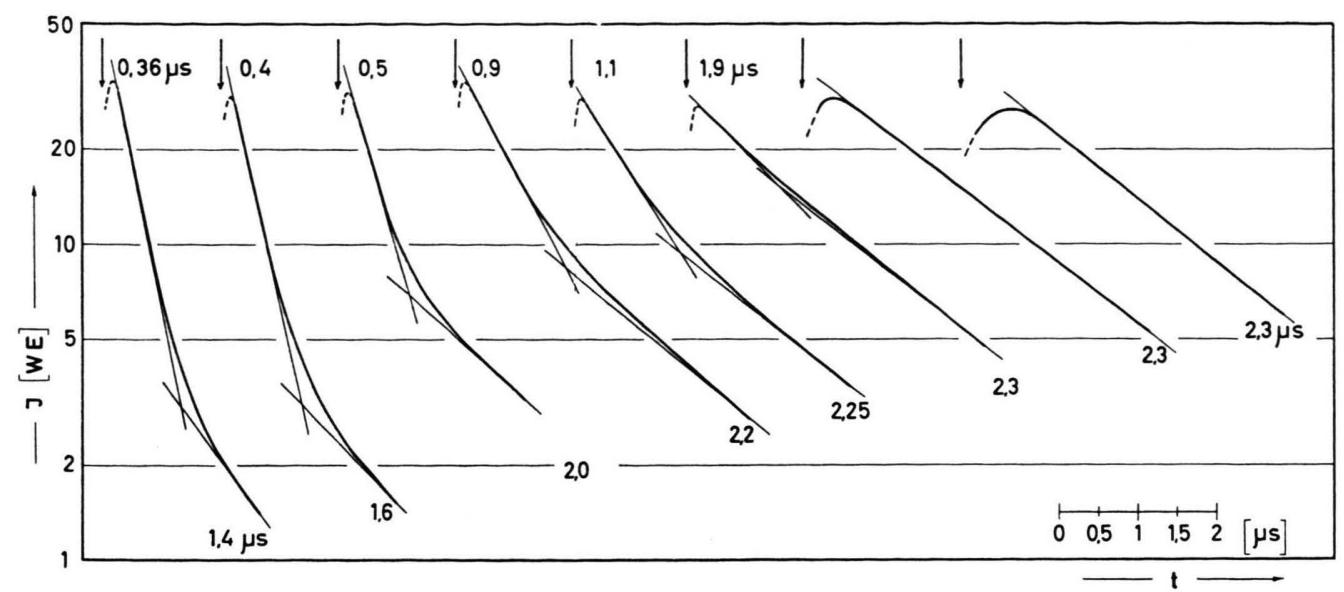

Abb. 2. Abklingkurven der ausgeleuchteten $371 \mathrm{~m} \mu$-Eigenlumineszenz bei $112{ }^{\circ} \mathrm{K}$ mit von links nach rechts abnehmender $\mathrm{V}_{\mathrm{K}}$-Zentrenkonzentration. Die Pfeile kennzeichnen den jeweiligen Zeitnullpunkt. Die Maximalintensität nimmt von der ersten Kurve bis zur letzten um etwa einen Faktor 50 ab. Zur Charakterisierung der Abklingkurven in Abhängigkeit vom Erregungszustand wurden „Anfangs“- und „Endgeraden“ mit den ihnen entsprechenden Zeitkonstanten eingezeichnet. Der noch vom ausleuchtenden Lichtblitz gestörte Teil der Abklingkurve wurde gestrichelt.

2. berichtete Abklingdauer $\tau$. Der Absolutwert von $\vartheta$ hängt von der Dauer der Röntgen-Erregung ab (Abb. 1).

4. Das Aussehen der Abklingkurven der ausgeleuchteten Lumineszenz und die Länge der Abklingzeit $\vartheta$ sind vom Erregungszustand des Kristalls abhängig: Nach einmaliger Röntgen-Vorbehandlung (ähnlich der unter 3 . beschriebenen) wurde aus $\mathrm{KJ}(\mathrm{In})$ bei $112{ }^{\circ} \mathrm{K}$ die in Abb. 2 gezeigte Folge von Lumineszenzabklingkurven aufgenommen. Der Erregungszustand der einzelnen Kurven wurde über die $\mathrm{V}_{\mathrm{K}}$-Zentrenkonzentration auf die Weise variiert, daß zwischen der Aufnahme einer Abklingkurve und der nächsten jeweils 2 Min. gewartet wurde. - Die Lumineszenz klingt bei großer $\mathrm{V}_{\mathrm{K}}$-Zentrenkonzentration zu Anfang mit der unter 2. berichteten Zeitkonstanten ab.

\section{Diskussion}

Die Tatsache, daß nach kurzer Röntgen-Bestrahlung aus $\mathrm{KJ}$ (In) Lumineszenz ausleuchtbar ist, weist darauf hin, daß analog zu den $\mathrm{Tl}^{+}$- auch die $\mathrm{In}^{+}$-Ionen Elektronen einfangen können. Ob die Elektronen durch das IR-Licht $(\lambda>850 \mathrm{~m} \mu)$ direkt ins Leitungsband gehoben werden oder nur in einen angeregten $\operatorname{In}^{\circ}$-Zustand gelangen, konnte nicht entschieden werden. Die Lebensdauer eines eventuellen angeregten $\mathrm{In}^{\circ}$-Zustands müßte jedoch im ganzen gemessenen Temperaturbereich mindestens um einen Faktor 5 kürzer sein als die des angeregten $\left\{\mathrm{V}_{\mathrm{K}}+\right.$ Elektron $\}$-Zustands ${ }^{\mathbf{1 1}}$.

Der zur Eigenlumineszenz gehörende angeregte $\mathrm{Zu}$ stand ist bei Ausleuchtung als $\left\{\mathrm{V}_{\mathrm{K}}+\right.$ Elektron $\}-\mathrm{Zu}$ stand ${ }^{3}$ und bei Erregung in der Grundgitterabsorption

11 H. Blume, Dissertation, Universität Freiburg 1967.

12 R. F. Wood, Phys. Rev. 151, 629 [1966].

13 Abschätzungen über die Lebensdauer freier Ladungsträger kann man z. B. gewinnen aus: G. R. Hugget u. K. TeE- als relaxiertes Exciton ${ }^{12}$ anzusehen. Unter der Voraussetzung, daß die Lebensdauer freier Elektronen vernachlässigbar kurz ist ${ }^{13}$, stellt die von uns gemessene Abklingdauer $\tau$ der ausgeleuchteten Lumineszenz des unverfärbten Kristalls die Lebensdauer des $\left\{\mathrm{V}_{\mathrm{K}}\right.$-Elek. tron\}-Zustands dar. Aus der Genauigkeit, mit der das exponentielle Abklingen der in der Grundgitterabsorption erregten Eigenlumineszenz nachgewiesen werden konnte, läßt sich abschätzen, daß die Lebensdauer eventuell freier, nicht lokalisierter Excitonen kurz im Vergleich zur Lebensdauer des relaxierten Excitonenzustands ist ${ }^{11}$. Aus der Gleichheit der Abklingdauern der ausgeleuchteten und der in der Grundgitterabsorption erregten Eigenlumineszenz folgt dann, daß die Lumineszenz bei beiden Erregungsarten dem gleichen elektronischen Übergang entspricht.

Die bei Ausleuchtung aus Kristallen, die durch RöNTGEN-Bestrahlung verfärbt waren, gemessenen Abklingzeiten (Abb. 1) können nach alledem keine Eigenschaft des Leuchtzentrums allein sein. Das Lumineszenzabklingen wird vielmehr durch eine verzögerte Elektronennachlieferung aus den Haftstellen verlängert.

Zur Deutung der Abklingkurven (Abb. 2) haben wir folgendes Modell entwickelt: Der Kristall enthält nach Röntgen-Bestrahlung im wesentlichen $\mathrm{V}_{\mathrm{K}}$-Zentren und mindestens zwei Sorten von Elektronenhaftstellen verschiedener Tiefe. Die flachen Haftstellen besitzen aufgefüllt einen gebundenen angeregten Zustand mit geringem thermischen Abstand sowohl zum Grundzustand wie auch zum Leitungsband. Die thermischen Abstände sind so klein, daß die Haftstelle auch unterhalb $120^{\circ} \mathrm{K}$ rasch entleert wird. Die tiefen Haftstellen [z. B. $\mathrm{In}^{+}$

garden, Phys. Rev. 141, 797 [1966] ; A. G. Redfield, Phys. Rev. 94, 537 [1954] ; R. K. Swank u. F. C. Brown, Phys. Rev. 130, 34 [1961]. 
oder $\mathrm{F}$ im $\mathrm{KJ}(\mathrm{In})$ ] enthalten Elektronen, die durch das ausleuchtende Licht ins Leitungsband gehoben werden. Diese Elektronen rekombinieren entweder mit $\mathrm{V}_{\mathrm{K}}$ Zentren - dabei entsteht unmittelbar Eigenlumineszenz -, oder sie werden vom angeregten Zustand der flachen Haftstellen eingefangen; dadurch kann nun verzögerte Eigenlumineszenz entstehen, wenn nämlich Elektronen aus dem angeregten Zustand der flachen Haftstellen $\mathrm{zu} \mathrm{V}_{\mathrm{K}}$-Zentren tunneln oder thermisch aus der Haftstelle wieder ins Leitungsband gelangen. Schließlich können Elektronen aus dem Leitungsband von tiefen Haftstellen eingefangen werden, wodurch sie für den Rekombinationsprozeß verlorengehen.

Die Annahmen des Modells werden insbesondere erzwungen durch den Satz Kurven für die Lumineszenzintensität (Ergebnis 4 und Abb. 2), die zunächst nur abklingen und allein durch Verringerung der $\mathrm{V}_{\mathrm{K}}$-Zentrenkonzentration in Kurven mit Anklingen übergehen. Das konnte nur durch die Annahme einer flachen Haftstelle mit angeregtem Zustand und durch die Einführung von Tunnelprozessen erreicht werden. - Ein Einfang der Elektronen durch tiefe Haftstellen muß berücksichtigt werden, sonst besäßen die Abklingkurven bei geringer $V_{K}$-Zentrenkonzentration keine feste Zeitkonstante (Abb. 2).

14 M. Hirai, M. Inezawa u. M. Ueta, J. Phys. Soc. Japan 17, 1483 [1962].

\section{Röntgen-Absorptionsspektren einiger Kohlenwasserstoffe im Bereich der $\mathrm{C}_{\mathrm{K}}$-Kante}

\author{
H.-U. Chun und H. Gebelein * \\ Institut für Physikalische Chemie der Universität \\ Frankfurt a. M.
}

(Z. Naturforschg. 22 a, 1813-1814 [1967] ; eingeg. am 9. Oktober 1967)

In einem vorangehenden Bericht ${ }^{1}$ über die langwellige Feinstruktur der Röntgen-Absorptionskante des Kohlenstoffs in seinen gasförmigen Verbindungen Methan, Äthylen, Benzol und Toluol teilten wir mit, daß eine deutliche Abhängigkeit der Lage ihrer Komponenten von der Art der Bindung des Kohlenstoffatoms besteht. Als Ursache der Feinstruktur der $\mathrm{C}_{\mathrm{K}}$-Kante nahmen wir, ausgehend von der Kosselschen Interpretation ${ }^{2}$ der RöNTGEN-Absorption, an, daß Übergänge eines ls-Elektrons in unbesetzte Molekülniveaus unterhalb seiner Ionisierungsgrenze (Absorptionskante) stattfinden. Eine nur bei den drei $\pi$-Elektronenverbindungen auftretende, sehr langwellige Absorptionslinie ordneten wir dem Übergang eines 1s-Elektrons in einen unbesetzten $2 \pi^{*}$-MO zu.

* Die mitgeteilten Meßresultate wurden im Rahmen der Diplomarbeit von H. Gebelein, Frankfurt a. M. 1967, erhalten.

1 H.-U. Chun, TAe-Kud Ha u. R. Mans, Z. Phys. Chem. [Frankfurt/M.] [N.F.] 47, 313 [1965].
Die das Modell beschreibenden reaktionskinetischen Differentialgleichungen sind unter der Annahme einer quasi-stationären Konzentration freier Elektronen im Leitungsband und der gleichen Annahme für die Konzentration der flachen Haftstellen im angeregten $\mathrm{Zu}$ stand integrierbar. Der so erhaltene analytische Ausdruck für die Lumineszenzintensität gestattet bei vernünftiger Wahl der Parameter, Kurven zu berechnen, die in sehr guter Übereinstimmung mit den gemessenen (Abb. 2) stehen, worüber in einer späteren Publikation ausführlicher berichtet wird.

Unsere Messungen und unsere Modellrechnung liefern in einer rohen Abschätzung für die Größenordnung der thermischen Tiefe der flachen Haftstelle 0,1 bis $0,2 \mathrm{eV}$. Daß derartig flache Haftstellen noch einen angeregten gebundenen Zustand haben können, läßt sich aus Arbeiten 14, 15 über F-Aggregatzentren mit einem zusätzlichen Elektron schließen.

Wir sind der Siemens-Aktiengesellschaft, Werner-Werk für Medizinische Technik, Erlangen, für ihre großzügige Unterstützung sehr zu Dank verpflichtet. - Auch der Deutschen Forschungsgemeinschaft haben wir für Unterstützung zu danken.

15 Shao-Fu Wang u. Chin Chu, Phys. Rev. 154, 838 [1967].
In Fortsetzung dieser Untersuchung wurden nach der früher beschriebenen Methode ${ }^{1,3,4}$ die RöntgenAbsorptionsspektren von Methan, Cyclohexan, Cyclohexen, Cyclohexadien-1,4, Cyclohexadien-1,3 und Benzol aufgenommen, um die mit dem Übergang von gesättigten über olefinische zu aromatischen Kohlenwasserstoffen verbundene Änderung der Feinstruktur der $\mathrm{C}_{\mathrm{K}}$-Kante eingehender zu verfolgen.

Die Abbildung gibt in der Form eines Termschemas eine Übersicht über die zu den einzelnen beobachteten Absorptionslinien gehörenden Energiewerte, die vom Molekülgrundzustand $(E=0)$ aus positiv gezählt sind. Die Fehlergrenze der Werte beträgt $\pm 0,1 \mathrm{eV}$.

Die Termschemata der untersuchten Kohlenwasserstoffe gliedern sich entsprechend der Natur der Verbindungen in drei Gruppen. Die gesättigten Kohlenwasserstoffe Methan und Cyclohexan besitzen eine sehr ähnliche Termfolge, desgleichen die olefinischen Verbindungen Cyclohexen und Cyclohexadien-1,4 sowie die Verbindungen mit konjugierten Doppelbindungen $\mathrm{Cy}$ clohexadien-1,3 und Benzol. Wie schon in der vorangehenden Untersuchung festgestellt wurde, tritt bei Anwesenheit von Doppelbindungen stets ein tiefliegender Term auf, der bei den gesättigten Verbindungen nicht beobachtet wird. Er entspricht, wie wir dort durch

2 W. Kossel, Z. Phys. 1, 124 [1920].

3 H.-U. Chun, Dissertation, Frankfurt (Main) 1963.

4 H. Hartmann u. H.-U. Chun, Theor. Chim. Acta 2, 1 [1964]. 